\title{
O Trabalho do Futuro? Motivações para o trabalho na economia compartilhada
}

\author{
Ursula A. S. Siqueira ${ }^{1, *}$, Carlos D. Santos ${ }^{2}$, \\ Paulo Roney Kilpp Goulart ${ }^{3}$
}

\begin{abstract}
http://orcid.org/0000-0002-5441-7456 / Universidade Federal do Pará (UFPA), Brasil
2http://orcid.org/0000-0001-5693-9795 / Universidade Federal do Pará (UFPA), Brasil

3 http:/ / orcid.org/0000-0001-5427-8743 / Universidade Federal do Pará (UFPA), Brasil
\end{abstract}

Os aplicativos de economia compartilhada impulsionaram um novo modelo de trabalho e um novo perfil de trabalhador. Considerando tal realidade, o presente estudo buscou identificar os principais motivadores de engajamento como trabalhador em plataformas de mobilidade urbana, mais especificamente de compartilhamento de carona (ridesharing). Em concomitância, investigou-se a influência de fatores socioeconômicos na relevância dos diferentes motivadores. Participaram da pesquisa quarenta e três motoristas de aplicativos, por meio online, a partir do aplicativo denominado Google Formulários. Para a análise de dados foi utilizado o modelo estatístico de regressão múltipla. Nos resultados encontrados, os motivadores considerados os mais relevantes para a escolha dos participantes foram Autonomia, Renda e Horário Flexível. Dos catorze motivadores investigados, dez apresentaram interações estatisticamente significativas com aspectos socioeconômicos. Os resultados também apontam diferenças entre os motivadores considerados como mais relevantes pelos participantes, quando comparados com estudos realizados em outros países. Salienta-se, portanto, a necessidade de um olhar diferenciado para a realidade brasileira.

Palavras-chave: trabalho, compartilhamento de carona, motivações.

\section{The work of the future? Work motivations in the sharing economy}

Abstract

Sharing economy applications have boosted a new work model and worker profile. The present study aimed to identify the main worker's motivators for engagement in urban mobility platforms, specifically in ridesharing applications. The influence of socioeconomic factors on the relevance of motivators was also investigated. Data was collected using an online survey from 43 drivers who work through applications. A multiple regression model was used for data analysis. The results showed that the most important motivators indicated by the participants were autonomy, income, and having flexible schedules. 10 out of the 14 motivators studied had significant interactions with socioeconomic aspects. The results also point out differences between the motivators considered most relevant by the participants, when compared to studies carried out in other countries. Therefore, a specific approach to this topic which is compatible with the Brazilian reality is needed.

Keywords: Work; Ridesharing; Motivations.

\section{¿El trabajo del futuro? Motivaciones para trabajar en la economía compartida}

\section{Resumen}

Los aplicativos de movilidad urbana impulsaron un nuevo modelo de trabajo y un nuevo perfil de trabajadores. Considerando tal realidad, este estudio buscó identificar los principales motivadores de la participación como trabajador en plataformas de movilidad urbana, más específicamente de viajes compartidos. En concomitancia, fue investigada la influencia de los factores socioeconómicos en la relevancia de los diferentes motivadores. Participaron de la pesquisa cuarenta y tres conductores de aplicativos, por medio on line, a través del aplicativo denominado Google Formulários. Para el análisis de datos, se utilizó el modelo estadístico de regresión múltiple. Según los resultados, los motivadores considerados más relevantes para los participantes fueron Autonomía, Ingresos y Horas Flexibles. De los catorce motivadores investigados, diez tuvieron interacciones estadísticamente significativas con aspectos socioeconómicos. Comparado con estudios realizados en otros países, los resultados mostraron diferencias entre los motivadores mas relevantes. Por lo tanto, se enfatiza la necesidad de una mirada diferente, ajustada a la realidad brasileña.

Palabras-clave: trabajo, viaje compartido, motivaciones. Trabalbo, 20(3), 1157-1165. https://doi.org/10.17652/rpot/2020.3.19659 
A economia compartilhada surgiu como um movimento alternativo ao hiperconsumo. Este novo modelo de negócios, caracterizado pelo compartilhamento de bens ou serviços, foi favorecido pelo aumento da demanda por modelos de produção sustentáveis. O compartilhamento neste molde se dá de várias formas e em diferentes modalidades de interação (Lutz, Newlands, \& Fieseler, 2018; Silveira, Petrini, \& Santos, 2016).

Os negócios classificados como de economia compartilhada possuem cinco características principais: são baseados em mercado, ou seja, criam e possibilitam a troca de bens ou serviços; possuem capital de alto impacto, devido as suas diversas possibilidades de criação de mercado de fácil inserção; são crowd-based, ou seja, o seu capital de trabalho vem de multidões de indivíduos, sendo descentralizados de instituições ou hierarquias; são baseados na troca entre pares, conectando diretamente o prestador a quem precisa do serviço, inclusive em situações que poderiam ser consideradas de cunho pessoal e passam a ser de cunho profissional (como dar carona); por último, possibilitam ocupações por demanda e sem a necessidade de um contrato formal de trabalho (Sundararajan, 2016).

Os serviços de mobilidade urbana, em específico, possibilitam a conexão entre usuários para a locomoção na cidade. Tais aplicativos, como a Uber, tiveram destacada expansão no Brasil (Mariano \& Diaz, 2017). Há ainda diferentes categorias dentro desta classificação de aplicação, podendo se tratar do compartilhamento de bicicletas (bikesharing), de veículos (carsharing) ou de caronas (ridesharing). Este último, caracterizado pelo serviço de condução de pessoas em automóveis de pequeno porte, nos quais se enquadram aplicativos como Uber, 99, Mobi, entre outros (Cohen \& Kietzmann, 2014).

De modo geral as empresas de economia compartilhada oferecem uma oportunidade de ocupação com flexibilidade de horários, de carga horária e de cronograma de trabalho. Diferente de empresas tradicionais, não há um contrato de remuneração fechado ou uma legislação que regulamente essas atividades, o que possibilita maior flexibilização (Li, Hong, \& Zhang, 2018).

Os trabalhadores ou prestadores que atuam em aplicativos de compartilhamento de carona encontram a mesma realidade. Plataformas dentro desta classificação funcionam como mediadoras que conectam passageiros a motoristas, objetivando a condução do primeiro a determinado local. Para a prestação de serviço como motorista, o trabalhador precisa utilizar um veículo pessoal e não-comercial, passando por uma verificação de informações pessoais. Ele é avaliado por cada corrida realizada e para se manter na plataforma precisa ter uma pontuação média mínima nessas avaliações (Raval \& Dourish, 2016).

Estudando os impactos dos aplicativos de compartilhamento de carona no mercado de trabalho dos EUA, a partir da entrada da Uber, Li et al. (2018) identificaram uma queda na taxa de desemprego e um aumento na força de trabalho. Observou-se também um aumento na demanda por trabalhadores para vagas de trabalho não qualificadas (low skill) em empresas tradicionais.

Uma grande parte dos estudos sobre engajamento em economia compartilhada utilizam questionários on-line para acessar informações. São exemplos Böcker e Meelen (2017), e Hawlitschek, Teubner e Gimpel (2016). O primeiro focou em usuários e provedores (prestadores de serviço) das modalidades de compartilhamento de carros, de carona, de acomodação, de alimentação e de ferramentas, considerando os motivadores econômicos, sociais e ambientais. Os resultados demonstraram que os prestadores em aplicativos de compartilhamento de carona seriam particularmente motivados por fatores sociais (Böcker e Meelen, 2017). O segundo estudo buscou identificar motivos contra e a favor da participação na economia compartilhada, mais especificamente na modalidade de carsharing, chegando a uma escala de 17 motivadores (Hawlitschek et al., 2016), escala esta que foi considerada no presente estu- do. É importante destacar que estes dois estudos não diferenciam o consumidor do prestador de serviço na plataforma (trabalhador).

Entretanto, a compreensão das motivações dos indivíduos está estritamente atrelada ao seu ambiente e, portanto, investigar os aspectos sociais que interferem nas motivações é importante (Verneque, Moreira, \& Hanna, 2012). Além disso, olhar para as especificidades do trabalho nesse modelo pode ampliar a compreensão das condições e impactos sobre a vida desses indivíduos e, subsidiariamente, da sociedade como um todo (Bucher, Fieseler, Lutz, \& Newlands, 2018).

Sendo assim, a proposta do estudo foi coletar dados especificamente do trabalhador engajado em aplicações de compartilhamento de carona, identificando variáveis socioeconômicas que poderiam ter influência sobre a escolha de engajamento. O objetivo geral foi identificar a relevância de diferentes motivadores para a participação como prestador em aplicações no Brasil, buscando investigar se fatores socioeconômicos exerceriam influência sobre eles.

Foram considerados os principais motivadores encontrados em pesquisas de motivação dentro da economia compartilhada. Após uma análise comparativa, foi possível identificar quais aspectos socioeconômicos foram mais determinantes para as diferentes relevâncias atribuídas às motivações desses trabalhadores.

\section{Método}

\section{Participantes}

Participaram da pesquisa um total de 52 prestadores em plataformas de economia compartilhada, dentre os quais 43 estavam trabalhando como motoristas em aplicativos de compartilhamento de carona e 9 eram hosts em plataformas de hospedagem. Visando garantir a qualidade da amostra estatística, foram consideradas as respostas apenas dos indivíduos que estavam trabalhando em aplicativos de compartilhamento de carona.

Para ser incluído na pesquisa, o participante deveria, portanto, estar trabalhando em uma plataforma de compartilhamento de carona atuante no Brasil, ser maior de 18 anos e obrigatoriamente concordarcomo Termo de Consentimento Livre e Esclarecido (TCLE).

\section{Instrumentos}

Em se tratando de um tema relativamente novo, não há ainda instrumento padronizado no que se refere a motivações para o trabalho dentro da economia compartilhada. A maioria dos estudos na área, utiliza o modelo de pesquisa survey (Fonseca, 2002), em caráter exploratório. Dentro dessa perspectiva, foi criado um instrumento específico para a realização da pesquisa, composto por dois questionários.

O primeiro questionário, teve como embasamento os resultados encontrados por Li et al. (2018) e Hawlitschek et al. (2016), sendo composto por 14 afirmações. Todas iniciavam com a frase "me interessei porque..." seguidas da descrição da variável motivadora (ver Tabela 1).

Sob uma perspectiva retroativa, o participante pontuava os motivadores para sua escolha. Para tanto, selecionava em uma escala do tipo Likert que ia de 1 a 10, sendo 1 equivalente a "não teve relação com minha decisão" e 10 "foi essencial para minha decisão".

O segundo questionário, socioeconômico, foi composto por 18 questões, em sua maioria com alternativas para escolha. As perguntas abertas requeriam respostas curtas e diretas, não interpretativas. Dentre as perguntas de múltipla escolha estavam idade, sexo, estado conjugal (alterado para status marital na análise), número de dependentes, região de residência, escolaridade, identificação das plataformas em que trabalhava, número de horas semanais 
Tabela 1

Definição dos quatorze motivadores considerados para a pesquisa e seu embasamento teórico, acompanhados de sua apresentação no questionário

\begin{tabular}{|c|c|c|}
\hline Motivador & Descrição & Referência no Questionário \\
\hline $\begin{array}{l}\text { Relatos de } \\
\text { Terceiros }\end{array}$ & $\begin{array}{l}\text { Variável social referente à influência da experiência de outras pessoas } \\
\text { na decisão de engajamento. }\end{array}$ & "Me interessei por conta dos relatos de sucesso de outras pessoas" \\
\hline $\begin{array}{l}\text { Experiência de } \\
\text { Relativos }\end{array}$ & $\begin{array}{l}\text { Variável social referente à influência do relacionamento com pessoas } \\
\text { que participam como prestadores nesse tipo de plataforma. }\end{array}$ & $\begin{array}{c}\text { "Me interessei porque conheci pessoas que estavam trabalhando com } \\
\text { esse tipo de plataforma" }\end{array}$ \\
\hline Publicidade & $\begin{array}{l}\text { Variável social referente à influência da mídia e publicidade realizada } \\
\text { pela plataforma. }\end{array}$ & "Me interessei por conta da publicidade realizada" \\
\hline Autonomia & $\begin{array}{l}\text { Não há uma hierarquia, sendo considerada uma relação P2P (baseado } \\
\text { na definição de Sundararajan, 2016). }\end{array}$ & "Me interessei pelo fato de não ser subordinado à ninguém" \\
\hline $\begin{array}{l}\text { Remuneração } \\
\text { Variável }\end{array}$ & $\begin{array}{l}\text { Dependente de produtividade e demanda (baseado no estudo de Li et } \\
\text { al., 2018). }\end{array}$ & "Me interessei porque minha remuneração depende de mim" \\
\hline Horário Flexível & $\begin{array}{l}\text { Cronograma e quantidade de horas dependem da escolha do provedor } \\
\text { (baseado no estudo de Li et al., 2018). }\end{array}$ & "Me interessei porque escolho meus horários e dias de trabalho" \\
\hline Anticapitalismo & $\begin{array}{c}\text { A crença de que essa forma de trabalho, baseada no compartilhamen- } \\
\text { to, seria uma forma de ir contra o capitalismo (baseado na escala de } \\
\text { Hawlitschek et al., 2016). }\end{array}$ & "Me interessei por acreditar ser uma forma de ir contra o capitalismo" \\
\hline $\begin{array}{l}\text { Satisfação em } \\
\text { Compartilhar }\end{array}$ & $\begin{array}{l}\text { Valorização do serviço como a possibilidade de compartilhar algo seu } \\
\text { com outros e ajudar pessoas (baseado no modelo de Hawlitschek et } \\
\text { al., 2016). }\end{array}$ & $\begin{array}{c}\text { "Me interessei pela possibilidade de compartilhar algo que é meu com } \\
\text { outras pessoas" }\end{array}$ \\
\hline Renda & $\begin{array}{l}\text { Utilização como uma forma de renda extra ou principal (baseado na } \\
\text { escala de Hawlitschek et al., 2016) }\end{array}$ & "Me interessei por conta do retorno financeiro" \\
\hline Conhecimento & $\begin{array}{l}\text { Escolha de engajamento por conhecer o modelo de economia compar- } \\
\text { tilhada (baseado na escala de Hawlitschek et al., 2016) }\end{array}$ & "Me interessei porque conheço esse modelo de compartilhamento" \\
\hline $\begin{array}{l}\text { Estilo de Vida } \\
\text { Moderno }\end{array}$ & $\begin{array}{l}\text { Crença de que este modo de trabalho representaria um estilo de vida } \\
\text { moderno (baseado no modelo de Hawlitschek et al., 2016). }\end{array}$ & $\begin{array}{c}\text { "Me interessei porque acredito que reflete um modelo de vida moder- } \\
\text { no" }\end{array}$ \\
\hline $\begin{array}{l}\text { Sensação de } \\
\text { Pertencimento }\end{array}$ & $\begin{array}{c}\text { A sensação de que a participação na plataforma o posiciona como } \\
\text { parte de uma comunidade que compartilha (baseado no modelo de } \\
\text { Hawlitschek et al., 2016) }\end{array}$ & $\begin{array}{l}\text { "Me interessei por me fazer sentir parte de uma comunidade que } \\
\text { compartilha" }\end{array}$ \\
\hline Experiência Social & $\begin{array}{l}\text { A possibilidade de interação social através do compartilhamento (base- } \\
\text { ado no modelo de Hawlitschek et al., 2016). }\end{array}$ & "Me interessei por poder conhecer e socializar com outras pessoas" \\
\hline Influência Social & $\begin{array}{l}\text { Crença de que a sociedade constrói uma estima por pessoas que com- } \\
\text { partilham (baseado no modelo de Hawlitschek et al., 2016) }\end{array}$ & $\begin{array}{c}\text { "Me interessei porque acredito que a sociedade aprecia quem compar- } \\
\text { tilha" }\end{array}$ \\
\hline
\end{tabular}

de dedicação ao trabalho, data de iniciação neste trabalho, quanto tempo intencionava permanecer no trabalho, renda individual e familiar antes da entrada e depois da entrada, se a plataforma era a principal fonte de renda e nível de satisfação com a plataforma. As únicas questões abertas eram referentes a ocupação antes de entrar para a plataforma e descrição de outras fontes de renda.

\section{Procedimentos de Coleta de Dados e Cuidados Éticos}

A pesquisa foi aprovada pelo comitê de ética do Núcleo de Medicina Tropical da UFPA. Os dados foram coletados on-line, através da ferramenta "Google Formulários" e enviados para sistema de nuvem acessado somente pelo pesquisador. $O$ formulário não requeria o e-mail do respondente, e não possibilitava identificar o computador ou celular utilizado para preenchê-lo. Portanto, o sigilo da identidade do participante foi garantido.

O respondente podia optar por receber os resultados da pesquisa, informando seu e-mail. A participação na pesquisa foi voluntária e só ocorria após a concordância com o TCLE, disponível na seção inicial do formulário. Foi garantido a todos os participantes o direito de desistir a qualquer momento, sem prejuízo para si ou para a pesquisa.

Para o recrutamento dos participantes a pesquisa foi divulgada em redes sociais diversificadas. O link de acesso aos questionários era acompanhado por um anúncio, resumindo os objetivos da pesquisa e perfil de participante procurado. Ao acessar o link, o participante visualizava o TCLE e selecionava se concordava ou não com a participação. Apenas em caso afirmativo ele era direcionado ao questionário de motivadores.

No início de cada questionário eram apresentadas orientações de como proceder para responder. Todas as questões eram obrigatórias, não sendo possível avançar sem as ter respondido.
Após responder o questionário de motivadores, o participante deveria clicar na opção "próximo" para prosseguir e era direcionado para o questionário socioeconômico. Ao finalizar e clicar em enviar, a tela de confirmação de participação era apresentada e sua resposta era automaticamente computada pelo sistema de nuvem.

\section{Procedimentos de Análise de Dados}

Primeiramente os dados foram separados por tipo de plataforma de engajamento, identificando-se a ocorrência de respondentes trabalhando em plataformas de transporte e hospedagem. Foram mantidas para análise apenas as respostas dos indivíduos engajados em plataformas de compartilhamento de carona, pois representavam a maior frequência de respondentes. Foi utilizado para a análise de dados o software estatístico R (R Core Team, 2018).

Para determinar a influência de fatores socioeconômicos sobre a relevância dos motivadores, foram utilizados modelos de regressão múltipla do tipo modelo linear generalizado (GLM). Essa análise estatística possibilita a determinação da relação entre uma variável dependente, com diferentes variáveis independentes ou preditoras, produzindo uma equação que pressupõe o melhor arranjo de variáveis preditoras para a previsão de uma variável dependente (Abbad \& Torres, 2002). Neste caso, foram construídos modelos de regressão múltipla para cada motivador, funcionando como variável dependente, relacionando-o com todos os fatores socioeconômicos em simultâneo, funcionando como variáveis preditoras.

Sendo que, a regressão múltipla requer um mínimo de observações por cada categoria das variáveis preditoras, categorias com poucas observações foram eliminadas ou agrupadas em categorias mais amplas. Este procedimento foi aplicado às seguintes variáveis: idade, escolaridade, ocupação anterior, renda individual anterior, renda familiar anterior, se possuía dependentes, status marital e se a plata- 
forma era a principal fonte de renda do respondente (ver Tabela 2).

Devido a amostra não ter apresentado distribuição normal, veram maior probabilidade de ser motivados do que participantes com salário superior (mais de 2 sal.). Por outro lado, o

Tabela 2

Categorização dos fatores socioeconômicos

\begin{tabular}{|c|c|c|}
\hline $\begin{array}{c}\text { Fator } \\
\text { socioeconômico }\end{array}$ & Categorização & Descrição \\
\hline \multirow{3}{*}{ Idade } & $18-29$ & Participantes que declararam ter entre 18 e 29 anos. \\
\hline & $30-40$ & Participantes que declararam ter entre 30 e 40 anos. \\
\hline & Mais de 40 & Participantes que declararam ter acima de 40 anos. \\
\hline \multirow{2}{*}{ Escolaridade } & EM & Participantes que declararam ter concluído o Ensino Médio completo ou abaixo. \\
\hline & ES & Participantes que declararam possuir Ensino Superior completo ou acima. \\
\hline \multirow{2}{*}{ Ocupação Anterior } & Desempregado & Participantes que declaram não ter nenhum vínculo empregatício antes da entrada para a plataforma. \\
\hline & Empregado & Participantes que declararam possuir vínculo empregatício antes da entrada para a plataforma. \\
\hline \multirow{2}{*}{$\begin{array}{l}\text { Renda Individual } \\
\text { Anterior \& Renda } \\
\text { Familiar Anterior }\end{array}$} & Até 2 sal. & Participantes que declaram renda (individual ou familiar) até 2 salários mínimos. \\
\hline & Mais de 2 sal. & Participantes que declaram renda (individual ou familiar) acima de 2 salários mínimos. \\
\hline \multirow{2}{*}{ Dependentes } & Com & Participantes que declararam possuir dependentes. \\
\hline & Sem & Participantes que declararam não possuir dependentes. \\
\hline \multirow{2}{*}{ Status Marital } & Casado & Participantes que declararam no item estado conjugal serem casados ou união estável. \\
\hline & Solteiro & Participantes que declararam no item estado conjugal estarem solteiros. \\
\hline \multirow{2}{*}{$\begin{array}{l}\text { Plataforma Principal } \\
\text { Fonte de Renda }\end{array}$} & $\operatorname{Sim}$ & Participantes que declararam que aderiram à plataforma como sua principal fonte de renda. \\
\hline & Não & Participantes que declararam que não aderiram à plataforma como sua principal fonte de renda. \\
\hline
\end{tabular}

a escala de motivação original foi transformada em binomial, ou seja, respostas entre 0 e 5 foram classificadas como "motivação reduzida" e respostas entre 6 e 10 foram classificadas como "motivação relevante". Consequentemente, na modelagem dos motivadores com base nas variáveis socioeconômicas foram utilizados modelos do tipo logístico, em que a variável dependente é binomial assumindo os valores 0 ou 1 (Abbad \& Torres, 2002).

Os modelos construídos para cada motivador foram simplificados pelo processo de stepwise, que exclui variáveis preditoras que tenham reduzida influência sobre a variável dependente (Abbad \& Torres, 2002). Este processo permite a construção de modelos que são menos complexos que os originais (denominados modelos reduzidos), mas que não são significativamente mais errôneos na tentativa de explicar os padrões de variação da variável dependente.

Para a apresentação dos resultados, as relações entre motivadores e variáveis socioeconômicas com significância estatística ( $p$-value menor que 0,05) foram graficadas utilizando o pacote effects do software R (R Core Team, 2018).

\section{Resultados}

Dentre os resultados obtidos, foi possível observar que os motivadores considerados como mais importantes para escolha dos participantes foram Autonomia, Renda e Horário Flexível, seguidos de Remuneração Variável, Experiência de Relativos, Experiência Social e Relato de Terceiros (Figura 1). Enquanto os motivadores pontuados como tendo menor relevância foram Publicidade, Conhecimento, Sensação de Pertencimento, Influência Social, Satisfação em Compartilhar e Anticapitalismo (Figura 1).

Dos 14 motivadores considerados, 10 apresentaram relações significativas com aspectos socioeconômicos. Dentre as principais variáveis preditoras encontradas estão características como idade, dependentes, status marital e escolaridade (Figuras 2, 3 e 4, Tabelas 3 e 4$)$.

O motivador Relato de Terceiros foi mais relevante para indivíduos que possuíam dependentes, assim como para casos em que o participante estava empregado quando optou por se engajar como prestador na plataforma (Figura 2, Tabela 4).

No caso do motivador Experiência de Relativos (Figura 2, Tabela 4), participantes com menor salário (até 2 sal.), ti- preditor idade demonstrou uma influência significativa sobre o motivador Remuneração Variável (Figura 2, Tabela 4), sendo este mais importante para os participantes mais jovens.

O motivador Autonomia teve como preditores mais significativos a idade e a escolaridade, sendo este um motivador mais importante para aqueles participantes de menor faixa-etária e que haviam concluído ao menos o ensino superior (Figura 3, Tabela 4).

Indivíduos que possuiam dependentes declararam maior influência do motivador Horário Flexível, quando em comparação com participantes sem dependentes (Figura 3, Tabela 4). Enquanto, no caso do motivador Renda, o preditor mais significativo foi a renda individual anterior, mostrando-se que era um motivador mais relevante para indivíduos que recebiam anteriormente menos que dois salários do que para indivíduos que recebiam mais do que dois salários.

$\mathrm{Na}$ Figura 4, podem ser observados os motivadores Conhecimento, Estilo de Vida Moderno e Experiência Social. Os três tiveram como preditor mais relevante o status marital. Em específico, respondentes casados foram mais propensos a considerá-los como relevantes. Ainda na mesma Figura 4 (Tabela 4), observa-se o motivador Sensação de Pertencimento associado a uma menor renda familiar antes do engajamento na plataforma.

Não foram encontradas relações significativas com os motivadores Publicidade, Anticapitalismo, Satisfação em Compartilhar e Influência Social. Estes também obtiveram menor pontuação na avaliação dos participantes. O motivador Conhecimento foi o único, dentre os considerados menos relevantes, que apresentou relação significativa com algum aspecto socioeconômico.

$\mathrm{Na}$ Tabela 3 é possível observar quais as variáveis preditoras foram excluídas dos modelos reduzidos pelo processo de stepwise. Na Tabela 4 são apresentados os parâmetros estatísticos dos modelos reduzidos para cada motivador.

\section{Discussão}

Os resultados encontrados demostram que aspectos socioeconômicos podem ter relação com as diferentes motivações de engajamento em atividades profissionais, como é o caso das plataformas de economia compartilhada. A maioria das pesquisas sobre motivação nessa área não faz essa rela- 


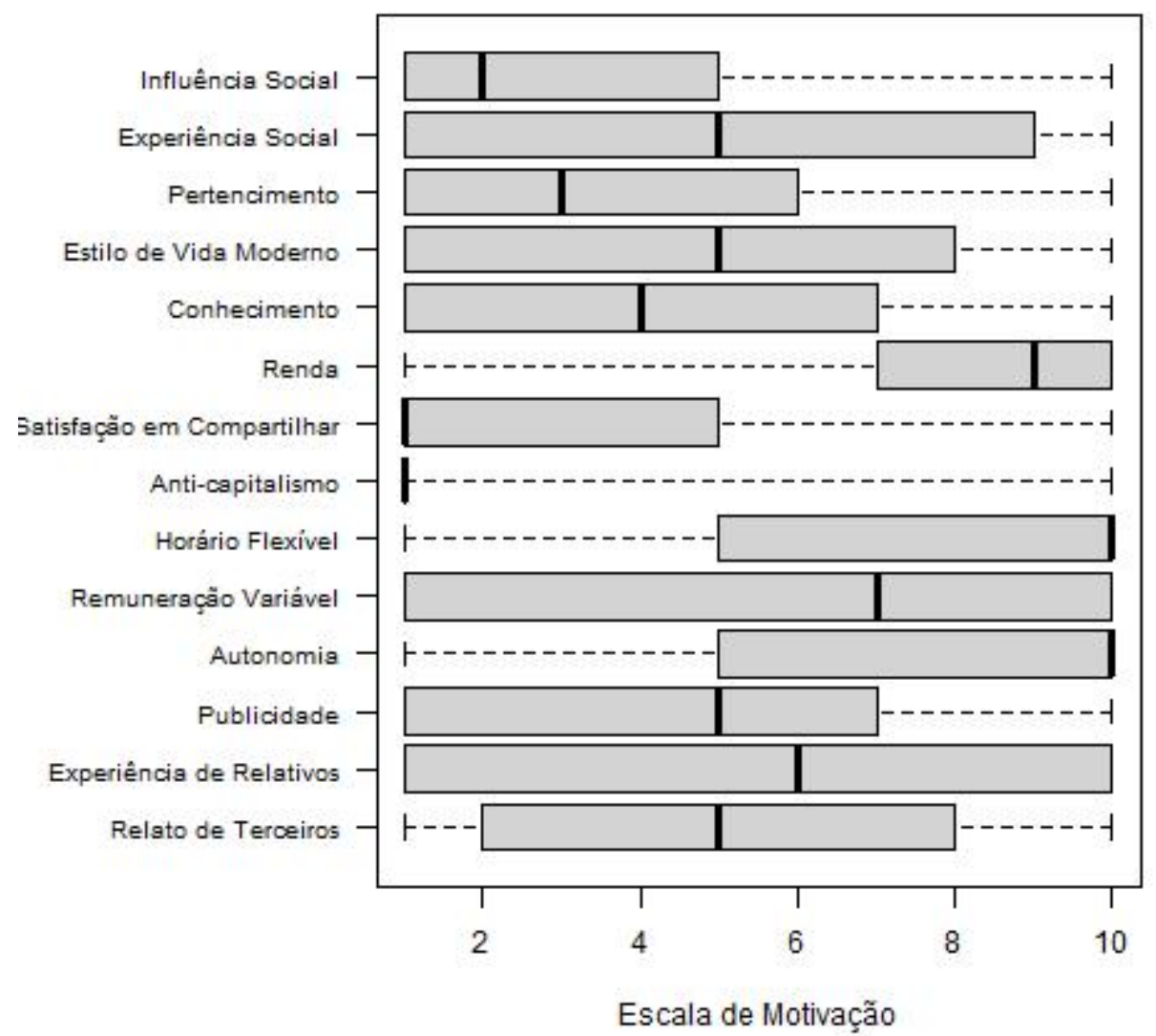

Figura 1. Escala de motivação de acordo com a pontuação atribuída pelos participantes. As caixas representam o intervalo das pontuações atribuídas pelos participantes para cada motivador. A linha preta dentro das caixas representa a mediana das respostas, a caixa estende-se entre o primeiro e o terceiro quartil e os bigodes estendem-se aos valores máximo e mínimo observados.

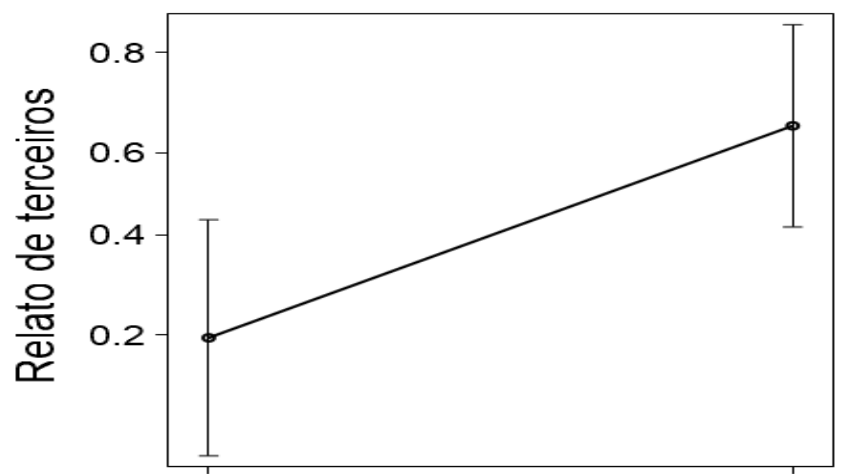

Desempregado Ocupação anterior

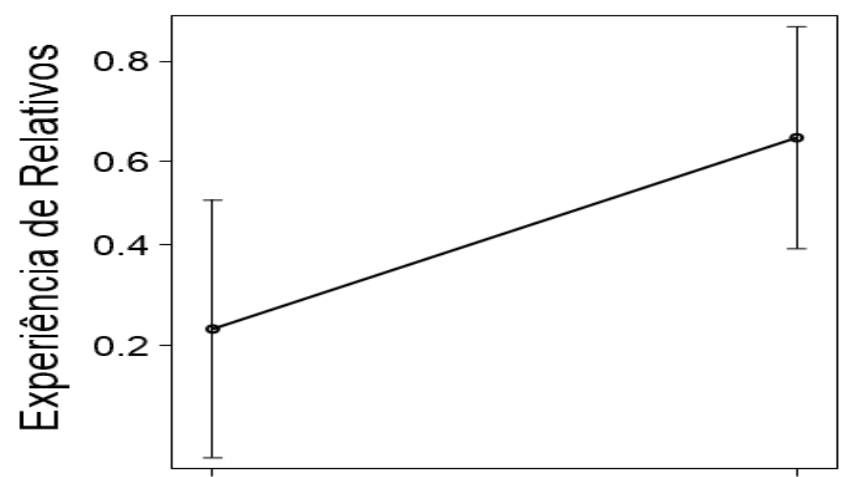

mais de 2 sal.

Renda Individual Anterior

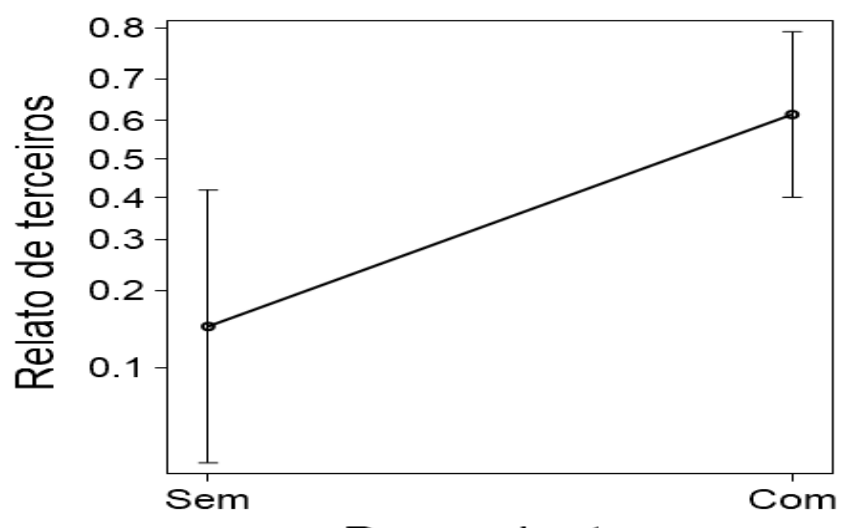

Dependentes

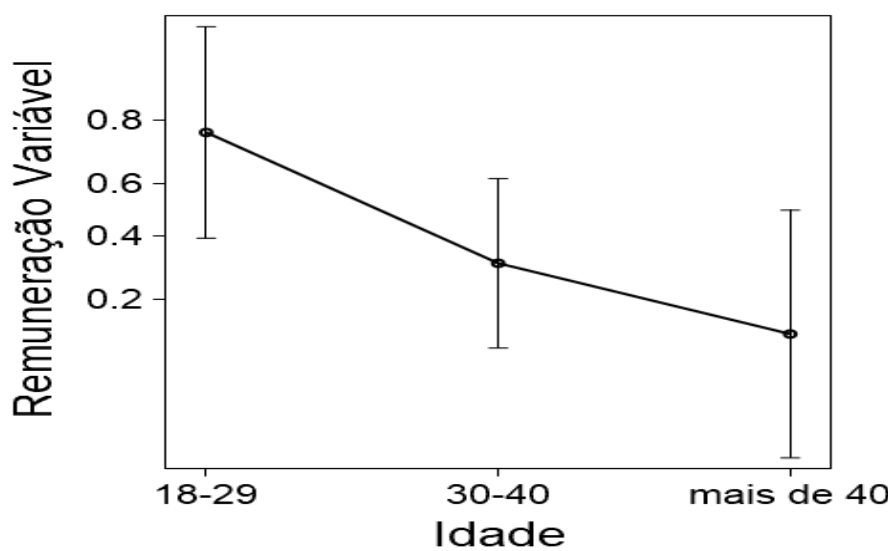

Figura 2. Gráficos de efeitos parciais dos modelos GLM relacionando os motivadores Relato de Terceiros, Experiência de Relativos e Remuneração Variável com variáveis socioeconômicas. Os valores na escala do y representam a probabilidade do respetivo motivador ser considerado relevante ou não para os participantes. As barras de erro no gráfico correspondem ao intervalo de confiança de $95 \%$. 

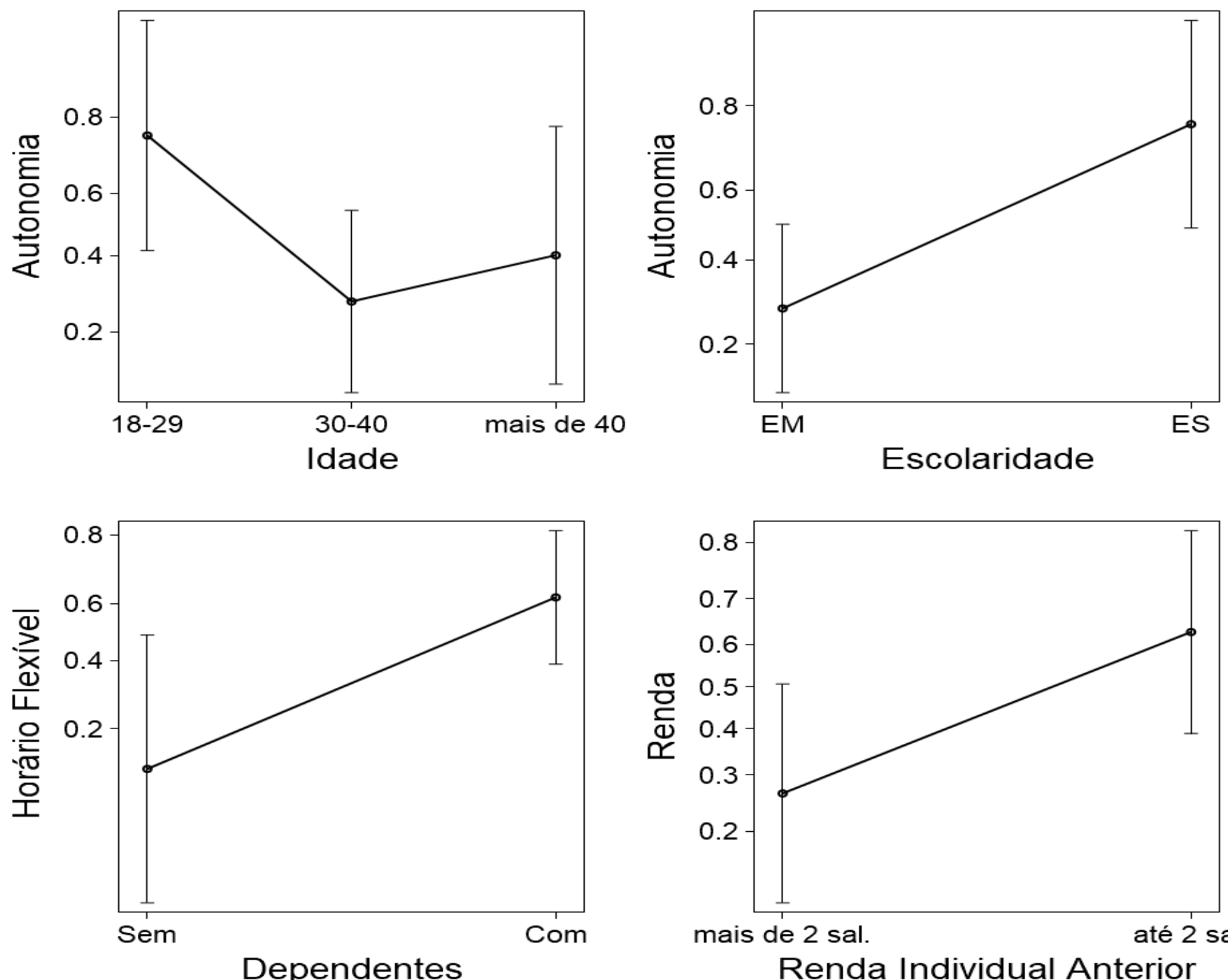

mais de 2 sal.

até 2 sal.

Renda Individual Anterior

Figura 3. Gráficos de efeitos parciais dos modelos GLM relacionando os motivadores Autonomia, Horário Flexível e Renda com variáveis socioeconômicas. Os valores na escala do y representam a probabilidade do respetivo motivador ser considerado relevante ou não para os participantes. As barras de erro no gráfico correspondem ao intervalo de confiança de $95 \%$.
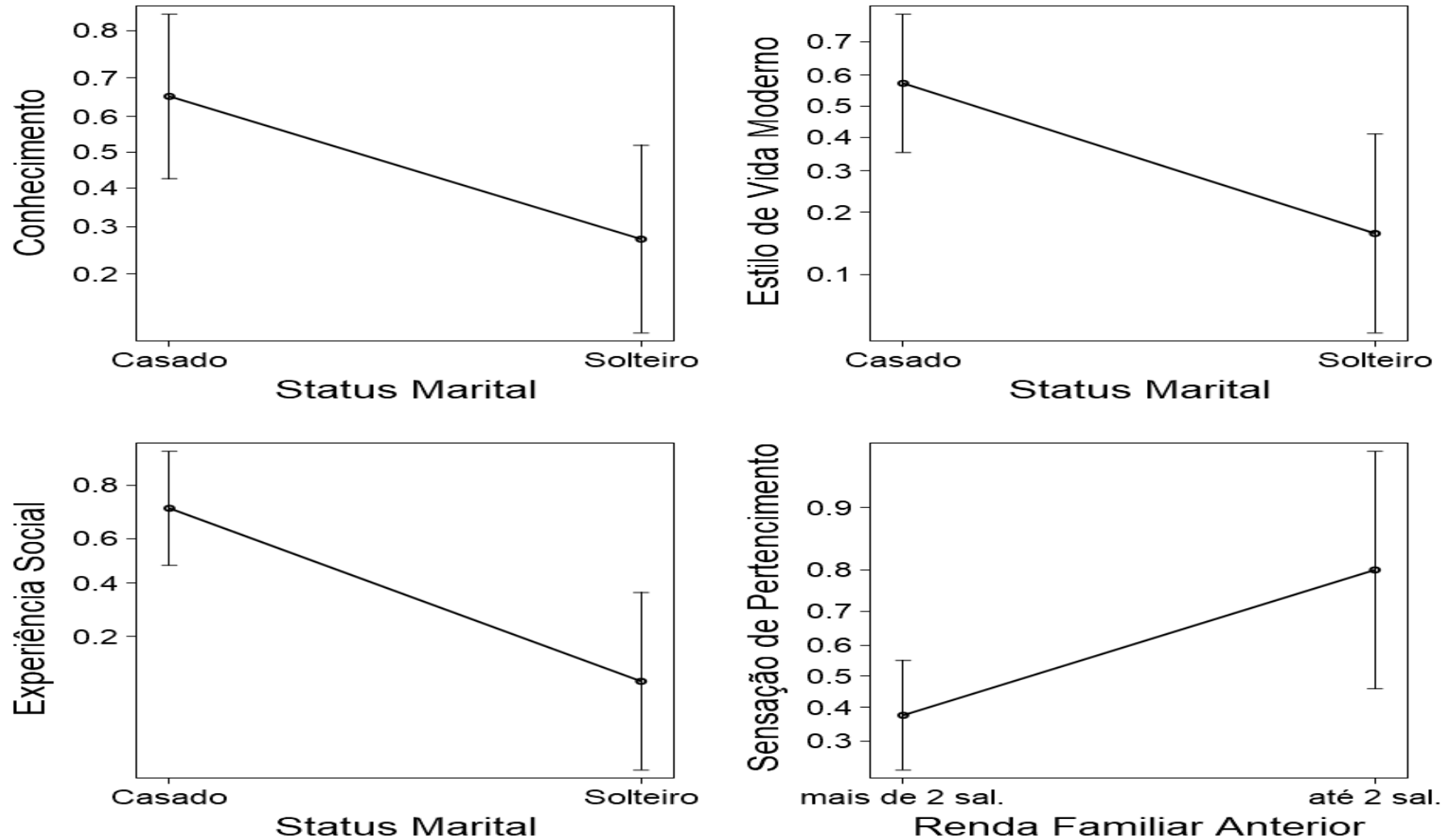
Tabela 3

Resumo da simplificação dos modelos por motivador através processo de stepwise.

\begin{tabular}{|c|c|c|c|}
\hline Motivador & Modelo & Preditores & AIC \\
\hline \multirow{2}{*}{$\begin{array}{l}\text { Relato de } \\
\text { Terceiros }\end{array}$} & Completo & $\begin{array}{l}\text { Idade, Status Marital, Dependentes, Escolaridade, Ocupação Anterior, Renda Ind. Anterior, Renda Familiar Anterior, Plata- } \\
\text { forma Renda Principal }\end{array}$ & 62,11 \\
\hline & Reduzido & Dependentes, Ocupação Anterior & 50,87 \\
\hline \multirow{2}{*}{$\begin{array}{l}\text { Experiência de } \\
\text { Relativos }\end{array}$} & Completo & Idade, Status Marital, Dependentes, Escolaridade, & 63,48 \\
\hline & Reduzido & Ocupação Anterior, Renda Ind. Anterior, Renda Familiar Anterior, Plataforma Renda Principal & 56,35 \\
\hline \multirow[t]{2}{*}{ Autonomia } & Completo & $\begin{array}{l}\text { Idade, Status Marital, Dependentes, Escolaridade, Ocupação Anterior, Renda Ind. Anterior, Renda Familiar Anterior, Plata- } \\
\text { forma Renda Principal }\end{array}$ & 63,56 \\
\hline & Reduzido & Idade, Dependentes, Escolaridade, Ocupação Anterior & 57,78 \\
\hline \multirow{2}{*}{$\begin{array}{l}\text { Remuneração } \\
\text { Variável }\end{array}$} & Completo & $\begin{array}{l}\text { Idade, Status Marital, Dependentes, Escolaridade, Ocupação Anterior, Renda Ind. Anterior, Renda Familiar Anterior, Plata- } \\
\text { forma Renda Principal }\end{array}$ & 68,56 \\
\hline & Reduzido & $\begin{array}{l}\text { Idade, Status Marital, Dependentes, Escolaridade, Ocupação Anterior, Renda Ind. Anterior, Renda Familiar Anterior, Plata- } \\
\text { forma Renda Principal }\end{array}$ & 68,56 \\
\hline \multirow{2}{*}{$\begin{array}{l}\text { Horário } \\
\text { Flexível }\end{array}$} & Completo & $\begin{array}{l}\text { Idade, Status Marital, Dependentes, Escolaridade, Ocupação Anterior, Renda Ind. Anterior, Renda Familiar Anterior, Plata- } \\
\text { forma Renda Principal }\end{array}$ & 68,02 \\
\hline & Reduzido & Idade, Dependentes, Renda Ind. Anterior & 58,63 \\
\hline \multirow{2}{*}{ Renda } & Completo & $\begin{array}{l}\text { Idade, Status Marital, Dependentes, Escolaridade, Ocupação Anterior, Renda Ind. Anterior, Renda Familiar Anterior, Plata- } \\
\text { forma Renda Principal }\end{array}$ & 65,27 \\
\hline & Reduzido & $\begin{array}{l}\text { Status Marital, Dependentes, Renda Ind. Anterior Ocupação Anterior, Renda Ind. Anterior, Renda Familiar Anterior, Plata- } \\
\text { forma Renda Principal }\end{array}$ & 57,44 \\
\hline \multirow{2}{*}{ Conhecimento } & Completo & Idade, Status Marital, Dependentes, Escolaridade, & 65,18 \\
\hline & Reduzido & Status Marital, Escolaridade, Renda Familiar Anterior & 54,83 \\
\hline \multirow{2}{*}{$\begin{array}{l}\text { Estilo de Vida } \\
\text { Moderno }\end{array}$} & Completo & $\begin{array}{l}\text { Idade, Status Marital, Dependentes, Escolaridade, Ocupação Anterior, Renda Ind. Anterior, Renda Familiar Anterior, Plata- } \\
\text { forma Renda Principal }\end{array}$ & 66,99 \\
\hline & Reduzido & Status Marital, Dependentes & 55,28 \\
\hline \multirow{2}{*}{$\begin{array}{l}\text { Experiência } \\
\text { Social }\end{array}$} & Completo & $\begin{array}{l}\text { Idade, Status Marital, Dependentes, Escolaridade, Ocupação Anterior, Renda Ind. Anterior, Renda Familiar Anterior, Plata- } \\
\text { forma Renda Principal }\end{array}$ & 57,43 \\
\hline & Reduzido & Status Marital, Dependentes & 49,62 \\
\hline \multirow{2}{*}{$\begin{array}{l}\text { Sensação de } \\
\text { Pertencimento }\end{array}$} & Completo & $\begin{array}{l}\text { Idade, Status Marital, Dependentes, Escolaridade, Ocupação Anterior, Renda Ind. Anterior, Renda Familiar Anterior, Plata- } \\
\text { forma Renda Principal }\end{array}$ & 65,84 \\
\hline & Reduzido & Renda Familiar Anterior & 56,35 \\
\hline
\end{tabular}

Nota. AIC = Akaike Information Criterion, valor que compara os modelos relativamente ao seu erro na tentativa de explicar os padrões de variação dos motivadores com base nas variáveis socioeconómicas. O modelo completo inclui todas as variáveis socioeconômicas como variáveis explicativas e o modelo reduzido apenas aquelas que têm uma influência significativa sobre o respectivo motivador.

ção, além de terem ocorrido majoritariamente em outros países.

Diferente dos resultados de alguns desses estudos ( $\mathrm{Li}$ et al., 2018; Böcker \& Meelen, 2017; Hawlitschek et al., 2016) o trabalhador engajado em plataformas de compartilhamento de carona no Brasil, demonstrou ser altamente motivado pelo aspecto econômico (Renda). Enquanto aspectos voltados para questões sociais e sustentáveis foram considerados como tendo menor importância na decisão de engajamento.

Isso corrobora com os resultados encontrados por Ferraz (2018). O estudo, realizado com jovens no estado de São Paulo, apontou que fatores sociais e sustentáveis não se caracterizavam como motivadores diretos da participação em plataformas de economia compartilhada. Contudo, segundo Prothero et al. (2011) ainda há a percepção de que o engajamento nessas aplicações estaria atrelado a questões sustentáveis.

Tais resultados podem ser reflexo de uma mudança cultural no que se refere a percepção do modelo. Quando surgiu, a economia compartilhada tinha como proposta uma forma alternativa de consumo, voltada para práticas coletivas e sustentáveis, que visavam a reutilização e colaboração (Lima, 2016). Porém, depois de já consolidada em países desenvolvidos, chegou ao Brasil com uma proposta de flexibilidade e autonomia no trabalho. A configuração de ganhos por produção e com poucas exigências de entrada, atreladas a um alto índice de desemprego (André, Da Silva, \& Nascimento, 2019), podem ter evidenciado o aspecto econômico.

No que concerne a flexibilidade de horário, Li et al. (2018), havia identificado este como um aspecto relevante para indi- víduos que não conseguiam trabalhar em horários fixos. Isso é observado nos resultados encontrados para o motivador Horário Flexível, mais significativo para pessoas que possuíam dependentes. Pode-se inferir que, participantes com atividades pessoais conflitantes com horários e carga de trabalho fixos, encontraram na economia compartilhada uma alternativa para conciliar sua vida pessoal com a profissional.

Outro dado a ser analisado é a relação entre o motivador Autonomia e o maior nível de escolaridade. Segundo Kaufman (2018), a autonomia é um fator importante para o bem-estar pessoal e, portanto, para o nível de satisfação dos indivíduos sobre suas vidas.

A hipótese de que indivíduos com maior nível educacional possuem mais motivações voltadas para a realização pessoal foi comprovada em um estudo que buscou a relação entre os níveis educacionais e a percepção de fatores motivacionais no trabalho, realizado por Ferreira, Demutti, e Gimenez (2010). A pesquisa mostrou que, enquanto pessoas com um nível de escolaridade menor tem motivações mais atreladas a aspectos voltados para a sobrevivência, indivíduos com maior nível escolaridade, principalmente com Ensino Superior, tendem a considerar como mais importantes motivações atreladas ao bem-estar pessoal.

Ainda em relação ao motivador autonomia, observouse que este foi considerado mais relevante pelos participantes mais jovens. O mesmo ocorreu quando analisado o motivador Remuneração Variável. Comprovadamente, pessoas mais novas mostram maior tendência de adesão a plataformas de economia compartilhada (Ferraz, 2018). Em específico, 
Tabela 4

Parâmetros estatísticos dos modelos reduzidos relacionando motivadores e variáveis socioeconômicas

\begin{tabular}{|c|c|c|c|c|c|}
\hline Motivador & Preditor & Beta & $E P$ & $Z$ & Valor- $p$ \\
\hline \multirow{2}{*}{ Relato de Terceiros } & Dependentes & 2,2384 & 0,8945 & 2,503 & 0,01233 \\
\hline & Ocupacao Anterior & 2,0821 & 0,8133 & 2,560 & 0,01046 \\
\hline \multirow{4}{*}{ Experiência de Relativos } & Ocupacao Anterior & 1,4700 & 0,7966 & 1,845 & 0,0650 \\
\hline & Renda Ind. Anterior & 1,8663 & 0,9461 & 1,973 & 0,0485 \\
\hline & Renda Familiar Anterior & $-1,9155$ & 1,0530 & $-1,819$ & 0,0689 \\
\hline & Plataforma Renda Principal & $-1,3621$ & 0,8222 & $-1,657$ & 0,0976 \\
\hline \multirow{4}{*}{ Autonomia } & Idade & $-2,1472$ & 1,0704 & $-2,006$ & 0,0449 \\
\hline & Dependentes & 2,0693 & 1,1310 & 1,830 & 0,0673 \\
\hline & Escolaridade & 2,1400 & 0,8416 & 2,543 & 0,0110 \\
\hline & Ocupação Anterior & 1,1957 & 0,7899 & 1,514 & 0,1301 \\
\hline \multirow{8}{*}{ Remuneração Variável } & Idade & $-3,1236$ & 1,5479 & $-2,018$ & 0,0436 \\
\hline & Status Marital & $-0,6224$ & 0,9665 & $-0,644$ & 0,5196 \\
\hline & Dependentes & 1,7953 & 1,2363 & 1,452 & 0,1465 \\
\hline & Escolaridade & 0,7999 & 0,7736 & 1,034 & 0,3011 \\
\hline & Ocupação Anterior & $-0,2151$ & 0,8928 & $-0,241$ & 0,8096 \\
\hline & Renda Ind. Anterior & -15669 & 1,1101 & -1412 & 0,1581 \\
\hline & Renda Familiar Anterior & 0,5939 & 1,2426 & 0,478 & 0,6327 \\
\hline & Plataforma Renda Principal & $-0,5932$ & 0,8430 & $-0,704$ & 0,4816 \\
\hline \multirow{3}{*}{ Horário Flexível } & Idade & $-2,4080$ & 1,2523 & $-1,923$ & 0,0545 \\
\hline & Dependentes & 2,4539 & 1,2483 & 1,966 & 0,0493 \\
\hline & Renda Ind. Anterior & $-1,3529$ & 0,7639 & $-1,771$ & 0,0766 \\
\hline \multirow{3}{*}{ Renda } & Status Marital & $-1,0447$ & 0,7524 & $-1,389$ & 0,1650 \\
\hline & Dependentes & 1,1914 & 0,8326 & 1,431 & 0,1525 \\
\hline & Renda Ind. Anterior & 1,5502 & 0,7678 & 2,019 & 0,0435 \\
\hline \multirow{3}{*}{ Conhecimento } & Status Marital & $-1,6256$ & 0,7290 & $-2,230$ & 0,0257 \\
\hline & Escolaridade & $-1,0802$ & 0,7495 & $-1,441$ & 0,1495 \\
\hline & Renda Familiar Anterior & 1,4933 & 0,9206 & 1,622 & 0,1048 \\
\hline \multirow{2}{*}{ Estilo de Vida Moderno } & Status Marital & $-1,9622$ & 0,8759 & $-2,240$ & 0,0251 \\
\hline & Dependentes & $-1,2178$ & 0,8867 & $-1,373$ & 0,1696 \\
\hline \multirow{2}{*}{ Experiência Social } & Status Marital & $-3,166$ & 1,125 & $-2,816$ & 0,00487 \\
\hline & Dependentes & $-1,627$ & 1,138 & $-1,429$ & 0,15289 \\
\hline Sensação de Pertencimento & Renda Familiar Anterior & 1,8971 & 0,8708 & 2,179 & 0,0294 \\
\hline
\end{tabular}

Nota. Beta = tamanho do efeito do preditor sobre o motivador; $E P=$ Erro Padrão associado a relação; $Z=$ estatística de teste da relação; Valor- $p=$ significância estatística da relação (valores inferiores a 0,05 representam relações significativas).

millenials (18-35 anos) geralmente buscam por independência e apresentam altas expectativas por recompensas pelo seu trabalho, aspecto aparentemente associado a altos níveis de autoconfiança (Ozcelik, 2015). Deste modo, é compreensível a tendência de que indivíduos mais jovens considerem Autonomia e Remuneração Variável como motivadores mais importantes.

Trata-se de uma forma alternativa de trabalho, com poucos critérios estabelecidos para que o indivíduo possa se tornar um prestador. Pessoas que normalmente estariam em vagas de baixa qualificação, acabam tendo a oportunidade de optar por melhores condições de trabalho, onde podem ganhar por produtividade, realizando tarefas relativamente simples (Li et al., 2018). Sendo assim, é compreensível que aqueles indivíduos com uma menor renda individual anterior atribuam uma alta relevância ao motivador Renda.

O compartilhamento em si, mesmo que não atrelado diretamente ao aspecto financeiro, pode ser considerado uma forma de aumento de capital social. Portanto, a sensação de conexão/pertencimento a um grupo tende a ser um motivador poderoso, podendo ser considerada uma estratégia de sobrevivência (Aunger \& Curtis, 2013). Olhando para a relação entre menor renda familiar e os motivadores Sensação de Pertencimento e Experiência de Relativos, pode-se fomentar uma reflexão quanto a se, poderia ser explicada como uma estra- tégia de sobrevivência, devido a uma possível falta de recursos.

Nos resultados encontrados também foi observada uma relação entre a ocupação anterior dos participantes e o motivador Relato de Terceiros. Aqueles indivíduos que estavam empregados antes de entrar na plataforma, foram mais propensos a declarar este como um motivador importante.

Considerando que pessoas desenvolvem suas relações com base nas recompensas e custos dessas interações, procurando manter relações que maximizem suas recompensas (Nawaz, Zhang, Mansoor, \& Ilmudeen, 2019), pode-se supor que pessoas empregadas anteriormente considerassem o relato de outros indivíduos como uma forma de maximizar a possibilidade de sucesso, sendo este um fator importante para que pessoas empregadas escolhessem se engajar neste modelo de trabalho.

Por último, um dos preditores que teve relação com diferentes motivadores, foi o status marital. Pessoas casadas mostraram-se mais propensas a considerar os motivadores Conhecimento, Estilo de Vida Moderno e Experiência Social, como relevantes. O dado parece sugerir que pessoas casadas são impulsionadas mais por aspectos relacionados ao impacto de suas atividades e a experiências que estas possam gerar, do que por questões relativas à renda.

Devido à grande quantidade de informações coletadas, não houve a possibilidade de aprofundamento na discussão de cada 
uma das interações. As relações discutidas entre motivadores e aspectos socioeconômicos/ambientais ilustram a relevância de uma abordagem contextual para uma visão mais ampla e informativa das motivações no trabalho. Estudo futuros poderão investigar de forma individualizada os motivadores, de modo a compreender melhor os aspectos contextuais que os influenciam.

Este momento de mudança nas configurações de trabalho derivadas da economia compartilhada (Li et al., 2018) abre espaço para novas pesquisas, que possam também trazer uma perspectiva contextualizada das motivações no trabalho. Ademais, é essencial refletir quanto às especificidades da realidade brasileira, entender que usuário e prestador são públicos diferenciados - portanto mais bem estudados separadamente - e reconhecer que existem especificidades do trabalho nos variados modelos de negócio da economia compartilhada. Tais aspectos, quando levados em consideração, tem muito a contribuir para a compreensão do novo perfil de trabalhador e o que podemos esperar dos modelos de trabalho daqui para frente.

\section{Referências}

Abbad, G., \& Torres, C. V. (2002). Regressão múltipla stepwise e hierárquica em Psicologia Organizacional: aplicações, problemas e soluções. Estudos de Psicologia, 7(SPE), 19-29. https://doi.org/10.1590/S1413-294X2002000300004

André, R. G., Da Silva, R. O., \& Nascimento, R. P. (2019). "Precário não é, mas eu acho que é escravo": Análise do Trabalho dos Motoristas da Uber sob o Enfoque da Precarização. Revista Eletrônica de Ciência Administrativa, 18, 7-34. https://doi.org/10.21529/RECADM.2019001

Aunger, R., \& Curtis, V. (2013). The anatomy of motivation : An evolutionary-ecological approach. Biol Theory, 8, 49-63. https://doi.org/10.1007/s13752$\underline{013-0101-7}$

Böcker, L., \& Meelen, T. (2017). Sharing for people, planet or profit? Analysing motivations for intended sharing economy participation. Environmental Innovation and Societal Transitions, 23, 28-39. https://doi.org/10.1016/j. eist.2016.09.004

Bucher, E., Fieseler, C., Lutz, C., \& Newlands, G. (2018). Managing Emotional Labor in the Sharing Economy. Academy of Management Proceedings, 2018(1), 1-40. https://doi.org/10.5465/AMBPP.2018.16933abstract

Cohen, B., \& Kietzmann, J. (2014). Ride on! Mobility business models for the sharing economy. Organization \& Environment, 27(3), 279-296. https://doi. org/10.1177/1086026614546199

Ferraz, C. D. S. (2018). Desvendando a economia colaborativa: um estudo quantitativo sobre as motivações de sua adesão (Dissertação de Mestrado, Escola Superior de Propaganda e Marketing, Brasil). Recuperado de http://tede2.espm.br/ handle/tede $/ 313$

Ferreira, A., Demutti, C., \& Gimenez, P. (2010). A teoria das necessidades de maslow: A influência do nível educacional sobre a sua percepção no ambiente de trabalho. Procedente de XIII SEMEAD (Seminários em Administração), Brasil, 2-17. Recuperado de https://d1wqtxts1xzle7.cloudfront. net/39156478/Teoria de Maslo.pdf?1444746247

Fonseca, J. J. S. (2002). Metodologia da pesquisa cientifica. Fortaleza, Brasil: UEC.

Hawlitschek, F., Teubner, T., \& Gimpel, H. (2016). Understanding the sharing economy - Drivers and impediments for participation in peer-to-peer rental. Proceedings of the Annual Hawaii International Conference on System Sciences, USA, 4782-4791. https://doi.org/10.1109/HICSS.2016.593

Kaufman, S. B. (2018). Self-Actualizing People in the 21st Century: Integration With Contemporary Theory and Research on Personality and Well-Being. Journal of Humanistic Psychology, O0(0), 1-30. https://doi. org/10.1177/0022167818809187

Li, Z., Hong, Y., \& Zhang, Z. (2018). An Empirical Analysis of the Impacts of the Sharing Economy Platforms on the U.S. Labor Market. Proceedings of the 51st Hawaii International Conference on System Sciences, USA, 666-674. Recuperado de http://hdl.handle.net/10125/49971

Lima, P. B. P. L. D (2016). Estilo de vida colaborativo: perfil e motivação dos usuários (Trabalho de Conclusão de Curso, Universidade Federal do Paraná, Brasil). Recuperado de http://hdl.handle.net/1884/50108

Lutz, C., Newlands, G., \& Fieseler, C. (2018). Emotional Labor in the Sharing Economy. Proceedings of the 51 st Hawaii International Conference on System Sciences, USA, 636-645. https://doi.org/10.24251/hicss.2018.081

Mariano, A. M., \& Diaz, L. F. A. (2017). A importância da aceitação e uso da tecnologia em aplicativos de mobilidade urbana: contribuições da literatura científica. Procedente do VII Congresso Brasileiro (CONBREPRO). Brasil, 1-12. Recuperado de https://www.researchgate.net/profile/Ari Mariano/ publication/321035342 A importancia da aceitacao e uso da tecnologia em aplicativos de mobilidade urbana contribuicoes da literatu- ra cientifica/links/5a09c17945851545eb594b00/A-importancia-da-aceitacao-e-uso-da-tecnologia-em-aplicativos-de-mobilidade-urbana-contribuicoes-da-literatura-cientifica.pdf

Nawaz, Z., Zhang, J., Mansoor, R., \& Ilmudeen, A. (2019). Gig workers in sharing economy: Conceptualizing Freelancer Value Proposition (FVP) in e-lancing platforms. Advances in Management and Applied Economics, 9(6), 5175. Recuperado de https://www.researchgate.net/profile/Zubair Nawaz3/ publication/334645068 Gig workers in sharing economy Conceptualizing Freelancer Value Proposition FVP in e-lancing platforms/links/ 5d37e202a6fdcc370a5a3a68/Gig-workers-in-sharing-economy-Conceptualizing-Freelancer-Value-Proposition-FVP-in-e-lancing-platforms.pdf

Ozcelik, G. (2015). Engagement and Retention of the Millennial Generation in the Workplace through Internal Branding. International Journal of Business and Management, 10(3), 99-107. https://doi.org/10.5539/ijbm.v10n3p99

Prothero, A., Dobscha, S., Freund, J., Kilbourne, W. E., Luchs, M. G., Ozanne, L. K., \& Thøgersen, J. (2011). Sustainable consumption: Opportunities for consumer research and public policy. Journal of Public Policy \& Marketing, 30(1), 31-38. https://doi.org/10.1509/ippm.30.1.31

R Core Team (2018). R: A language and environment for statistical computing. Vienna, Austria: R Foundation for Statistical Computing.

Raval, N., \& Dourish, P. (2016). Standing out from the crowd: Emotional labor, body labor, and temporal labor in ridesharing. Proceedings of the 19th ACM Conference on Computer-Supported Cooperative Work \& Social Computing, USA, 97-107. https://doi.org/10.1145/2818048.2820026

Silveira, L. M., Petrini, M., \& Santos, A. C. M. Z. (2016). Economia compartilhada e consumo colaborativo: o que estamos pesquisando? REGE - Revista de Gestão, 23(4), 298-305. https://doi.org/10.1016/i.rege.2016.09.005

Sundararajan, A. (2016). The sharing economy: The end of employment and the rise of crowd-based capitalism. Cambridge, MA: Mit Press.

Verneque, L., Moreira M. B., \& Hanna, E. S. (2016). Motivação. Em F. D. M. Silvares, F. B. Assumpção Junior, \& L. Priszkulnik (Eds.), Temas clássicos da psicologia sob a ótica da análise do comportamento (pp. 74-87). Rio de Janeiro: Guanabara Koogan.

\section{Informações sobre os autores:}

\section{Ursula A. S. Siqueira}

Núcleo de Teoria e Pesquisa, Prédio II, sala 22, Campus

Universitário do Guamá. Rua Augusto Corrêa, 01, CEP 66075-

110, Guamá (Belém - PA), Brasil.

E-mail: ursula.s.siqueira@outlook.com

\section{Carlos D. Santos}

E-mail: cdsantos@ufpa.br

\section{Paulo Roney Kilpp Goulart}

E-mail: goulart@ufpa.br 